\title{
Silencing microRNA155 transforms the properties of endotoxin tolerant dendritic cells and enhance its therapeutic effect on sepsis murine model.
}

\section{Min Yang}

Ningbo City First Hospital

\section{Chao-Chen Hou}

The First Affiliated Hospital of Wenzhou Medical University

\section{Yan-Yan Yang}

The First Affiliated Hospital of Wenzhou Medical University

Shan-Shan Li

The First Affiliated Hospital of Wenzhou Medical University

\section{De-Yong Kong}

The First Affiliated Hospital of Wenzhou Medical University

\section{You-Ran Chen}

The First Affiliated Hospital of Wenzhou Medical University

\section{Hui-Fang Zhang}

The First Affiliated Hospital of Wenzhou Medical University

\section{Ming-Qin Lu ( $\nabla$ Imq0906@163.com )}

Department Of Infection And Liver Diseases,Liver Reasearch Center,The First Affiliated Hospital Of Wenzhou Medical University ,Wenzhou,Zhejiang,China https://orcid.org/0000-0002-8331-5862

\section{Research article}

Keywords:

Posted Date: October 8th, 2020

DOl: https://doi.org/10.21203/rs.3.rs-86685/v1

License: (c) (i) This work is licensed under a Creative Commons Attribution 4.0 International License. Read Full License 
Silencing microRNA155 transforms the properties of endotoxin tolerant dendritic cells and enhance its therapeutic effect on sepsis murine model.

Authors: Min Yang ${ }^{1 *}$,Chao-Chen Hou ${ }^{2 *}$, Yan-Yan Yang ${ }^{2}$, Shan-Shan Li $^{2}$, De-Yong Kong $^{2}$, You-Ran Chen ${ }^{2}$, Hui-Fang Zhang ${ }^{2}$, Ming-Qin Lü

Institution: ${ }^{1}$ Department of Ultrasound, Ningbo First Hospital, Ningbo, Zhejiang, China. ${ }^{2}$ Department of Infection and Liver Diseases, Liver Research Center, the First Affiliated Hospital of Wenzhou Medical University, Wenzhou, Zhejiang, China.

*Both authors contributed equally.

\#Corresponding Author

Correspondence: Dr Ming-Qin Lu, Department of Infection and Liver Diseases, Liver Research Center, the First Affiliated Hospital of Wenzhou Medical University, Wenzhou 325000, Zhejiang, China.

Tel: +86 577555 79622; fax: +86577880 78262;

e-mail:lmq0906@163.com 


\section{Abstract}

Sepsis refers to the body's dysfunctional response to infection and leads to lifethreatening organ dysfunction. Dentritic cells (DCs), a kind of powerful functional antigen-presenting cell, plays a critical role in immune response and has been used in the field of immunotherapy for multiple diseases. Endotoxin tolerance is a phenomenon that believed to prevent the organism from producing persistent and excessive inflammatory reaction and numerous studies indicated that endotoxin tolerant dendritic cells (ETDCs) have therapeutic effects on experimental models of several diseases. MicroRNA155(miR155), which plays a pivotal role in various physiological and pathological processes in immunity, was reported associated with the formation of endotoxin tolerance. In this paper, we explored the change of properties after silencing miR155 in ETDCs then attempted to study its effects on the murine model of sepsis. Endotoxin tolerant dendritic cells were transfected with adenovirus silencing miR155 and negative control adenovirus, thereafter collected for reverse transcription polymerase chain reaction of miR155, costimulatory molecules analysis, allogeneic mixed lymphocyte reaction and cytokine concentration evaluation. 42 balb/c mice were randomly divided into control group, sham operation group, sepsis group, ETDCs 
treatment group and miR155/-ETDCs treatment group. Two treatment groups respectively received tail vein injection of ETDCs and miR $155^{-/}$ETDCs $24 \mathrm{~h}$ earlier than cacal ligation puncture. Our results revealed that silencing miR155 could deepen the immature status of ETDCs and both ETDCs and miR155/-ETDCs performed a protective effect in murine sepsis models while miR $155^{-/}$ETDCs got a better protection. We reasoned that miR155 participates the formation of endotoxin tolerance and the protective effects of ETDCs and miR $155^{-/}$ETDCs in the early stage of sepsis may achieved by altering the concentration of mouse cytokines to affect $\mathrm{T}$ cell differentiation and exert negative immune regulation.

Keywords:endotoxin tolerance, microRNA155, sepsis

\section{Backgroud}

Sepsis refers to a syndrome of physiologic, pathologic, and biochemical abnormalities induced by infection, leads to life-threatening organ dysfunction ${ }^{1}$. Characterised by a dysfunctional host immune response comprising both proinflammatory and anti-inflammatory or immunosuppressing components, sepsis affects all types of immune cells and their compartments ${ }^{2}$. Despite a variety of clinical treatment measures, sepsis remains a major health problem worldwide, associated with high mortality rates in all countries ${ }^{3}$. Compelling evidence indicate that the derailment from immune homeostasis underlies sepsis-associated acute and long-term mortality ${ }^{4,5}$. Therefore, there is an emergency to seek new strategies to target immune deficiency in 
sepsis to improve the prognosis. Considering sepsis involving in complex network of proinflammatory and anti-inflammatory cytokines ${ }^{6}$, specific targeting a single mediator maybe not a ideal choice. Recent years, adoptive cellular immunotherapy gradually has become a research hotspot.

Dentritic cells (DCs), a kind of powerful functional antigen-presenting cell, plays a critical role in mediating innate immune response and inducing adaptive immune response $^{7}$, has been used in the field of immunotherapy for diseases such as autoimmune diseases, malignant tumors, and parasitic infections ${ }^{8}$.Endotoxin tolerance is a phenomenon that prior exposure of innate immune cells like monocytes/macrophages to minute amounts of endotoxin cause them to become refractory to subsequent endotoxin challenge ${ }^{9}$.The formation of endotoxin tolerance prevents the organism from producing persistent and excessive inflammatory reaction. Our previous study indicated that the utilization of endotoxin tolerance could attenuate experimental acute liver failure ${ }^{10,11}$.Similarly, ample studies has proved endotoxin tolerant dendritic cells (ETDCs) have therapeutic effects on experimental models of several diseases ${ }^{12-14}$. Although endotoxin tolerance has attracted lots of attention these years, the biological machanism remains elusive.

MicroRNAs(miRNAs) is evolutionarily conserved non-coding small RNAs that range from 18 to 25 nucleotides (nt) in length ${ }^{15}$, participates in the fine-tuning of many fundamental biological processes ${ }^{16}$. As one of the best characterized miRNAs, microRNA155(miR155) plays a pivotal role in various physiological and pathological processes such as immunity, inflammation, viral infections, cancer, cardiovascular 
disease, hematopoietic lineage differentiation, and Down syndrome ${ }^{17}$. miR155's uniquely expressed in activated cells of the immune system while its deficiency can cause serious defects in immune function ${ }^{18}$. Doxaki et $\mathrm{al}^{19}$ had demonstrated that miR155 is associated with the formation of endotoxin tolerance. But the current understanding of how miR155 affects endotoxin tolerance and sepsis still be rudimentary.

In this study,we investigated the change of properties after silencing miR155 in ETDCs then attempted to explore its effects on the murine model of sepsis.

\section{Materials and Methods}

\subsection{Animals}

Male balb/c and c57bl/6 mice aged 6-8 weeks were purchased from the Shanghai Laboratory Animal Center (Shanghai,China) and housed under specific pathogen-free conditions in the Experimental Animal Laboratory of Wenzhou Medical University (Wenzhou, Zhejiang, China).All mice had free access to standard laboratory water and chow and were treated in accordance with the Guide for the Care and Use of Laboratory Animals of the National Institutes of Health. The research protocol was approved by the Animal Ethics Committee of Wenzhou Medical University.

\subsection{Reagents}

Adenovirus silencing miR155 (aden-miR155 $5^{-/}$) and negative control adenovirus (aden-con) were constructed by Hanbio (shanghai,China). Lipopolysaccharides (L4391-1mg) was purchased from Sigma Chemical (St.Louis,MO,USA). 
Recombinant mouse granulocyte-macrophage colony stimulating factor (rmGM-CSF) and Interleukin-4 (IL-4) were purchased from PeproTech CO. (London, UK). Murine tumor necrosis factor-alpha(TNF- $\alpha$ ) and interleukin-10 (IL-10) enzyme-linked immunosorbent assay(ELISA) kits as well as mouse regulatory $\mathrm{T}$ cellstaining kit, mouse Th17 staining kit were purchased from the MultiSciences (Hangzhou,China). PE-,Percp-cy5.5 or APC- conjugated mouse mAb to MHC-II (IA), CD86 and CD11c Cell Proliferation DyeeFluor 670 were purchased from eBioscience CO. (SanDiego, CA). Cell Counting Kit 8 was abtained from Beyotime Biotechnology CO.(Shanghai,China).The isoflurane vaporizer was purchased from Matrx; Midmark Corp., Dayton (OH, USA).

2.3 BMDCs preparation and the induce of ETDCs

Bone marrow-derived DC cells (BMDCs) were isolated from femurs and tibias of male balb/c mice, resuspended in RPMI 1640 medium (Gibco, USA) containing 10\% fetal bovine $\operatorname{serum}(\mathrm{Gibco})$ and $1 \%$ penicillinstreptomycin mixture, seeded in 6-well tissue culture plates and left to adhere for $8 \mathrm{~h}$ at $37^{\circ} \mathrm{C}$ in a humidified $5 \% \mathrm{CO} 2$ atmosphere. Then BMDCs were cultured with RPMI 1640 medium supplemented with $10 \%$ FBS in the presence of 20ng/ml rmGM-CSF and 10ng/ml IL-4 (PeproTech, USA). All of the culture supernatants were replaced with new medium mixed with cytokines in day2. We changed half medium and add cytokines every two days. BMDCs were plated in a 6-well plate and add LPS(100ng/ml) into culture supernatants to induce ETDCs at day4.

2.4 adenovirus transfection 
According to different treatments, all cells were separated into five groups named imDCs,mDCs(DCs+large dose LPS), ETDCs(ETDCs+ large dose LPS), ETDCs-con(ETDCs+ negative adenovirus + large dose LPS), miR155 ${ }^{-}$ETDCs $\left(\right.$ miR155 ${ }^{-}$ETDCs + high dose LPS). At day5,the ETDCs were transfected with adenmiR155 ${ }^{-/}$and aden-con at an appropriate multiplicity of infection(MOI) for 6 hours in RPMI1640 with 10\%FBS.Subsequently the culture supernatants containing virus suspension was replaced by new medium mixed with IL-4 and rmGM-CSF. After stimulated by large doses of LPS $(10 \mathrm{mg} / \mathrm{ml})$ at day7 for $24 \mathrm{~h}$,all groups of cells were collected for subsequent experiments.

2.5 RNA isolation and qRT-PCR analysis

After endotoxin tolerance induction,total RNA was extracted from culture cells using TRIzol regent (Life technologies) according to the manufacturer's instructions. Total microRNA was isolated from DCs using a miRcute miRNA isolation kit (TiangenBiotech,Beijing China) under manufatrer's guidance. Whereafter, we used a RevertAidFirstStand cDNA Kit(Thermo Fisher) to reverse-transcribe total RNA to cDNA. The cDNAs were subjected to realtime PCR analysis using a ABI7000 prism Real-time System (Thermo Fisher) with a Power SyberGreen PCR Master Mix (Takara).MiR-155 primersfor RT-PCR were designed by Haofeng(Wenzhou, China). 2.6 Mixed Lymphocyte Reaction(MLR) and analysis of T cells proliferation Splenic $\mathrm{CD}^{+} \mathrm{T}\left(2 \times 10^{4} /\right.$ well $)$ cells purified from c57bl /6 mice were plated in a 96-wells plate. All DCs were preteated with mitomycin C(MCE,NJ,USA) for half hour and then washed with PBS twice. Each group pf DCs and T cells were cultured in two 
different ratios of 1:5 and 1:10 for 4 days subsequently. Cell Counting Kit(MCE,NJ,USA) 10 $\mu 1$ was added into culture medium, after 4 hour incubation, all 96wells plates were detected by microplate reader(Bio-rad).

\subsection{Animal models}

$42 \mathrm{balb} / \mathrm{c}$ mice were randomly divided into control group(6), sham operation

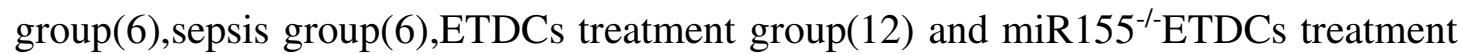

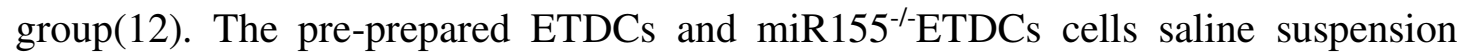
$\left(1 \times 10^{7} / \mathrm{ml}\right)$ was reinfused through caudal vain to the mice in ETDCs treatment group and $\mathrm{miR} 155^{--}$ETDCs treatment group respectively $(0.2 \mathrm{ml}$ each mouse) while sepsis group mice were injected with same volume $0.9 \%$ saline. Mice in sepsis group, ETDCs treatment group and $\mathrm{miR} 155^{--}$ETDCs treatment group were anesthetized by inhalation of isoflurane with an isoflurane vaporizer and established sepsis model via cacal ligation puncture (CLP) after 24h. Sham operation group was performed with simple abdominal incision and saturation while control group did not receive any treatment.

2.8 Cytokine analysis

The cell supernatant of each group and serum of each group mice were collected. The concentrations of IL-10 and TNF- $\alpha$ of each group were examined using ELISA kit by manufactrer's instruction.

2.9 Flow cytometry analysis

The cells of five groups were harvested and stained with PE-, PEcy7- or APCconjugated anti-mouse MHC-II, CD86 and CD11c Abs. The spleen T lymphocytes $\left(1 \times 10^{7} / \mathrm{ml}\right)$ of each group mice were isolated after execution. $100 \mu \mathrm{l}$ cell suspension was 
stained with CD4,FITC and CD25,APC followed by incubation of Foxp3,PE, while another $100 \mu \mathrm{l}$ stained with CD4,PerCP-Cy5.5 and IL-17A, PE. All cells were fixed with $1 \%$ paraformaldehyde and analyzed by flow cytometry.

\subsection{Histopathological analysis}

After sacrificing all the mice 24 hours after the operation, the liver, kidney and ileal tissue of each group were taken and made into pathological section. Hematoxylin-eosin (HE) staining was performed to visualize the pathological changes.

\subsection{Statistical analysis}

All data are presented as means \pm standard deviation (SD). One-way analysis of variance (ANOVA) and the least significant difference (LSD) test in SPSS19.0 software were used to analyze the statistical differences among multiple groups. A value of $\mathrm{P}$ less than 0.05 was considered statistically significant $(\mathrm{P}<0.05)$, each result was repeated at least three times.

\section{Results}

3.1 successful transduction of aden-miR155 $5^{-/}$inhibited miR155 expression

Previous studies have confirmed that recombinant adenovirus is a efficient vehicle for DC gene transfer ${ }^{20}$. Adenovirus-miR $155^{-/}$and control adenovirus were transfected into ETDCs respectively, thereafter we observed the cells with inverted fluorescence microscope and photographed the fluorescent images, confirming that the transfection rate exceeded 85\%.(Fig.1) The expression of miR155 in ETDCs 
transducted with aden-miR $155^{-/-}$were significantly decreased, approved that the aden-miR155 ${ }^{-/-}$inhibit the expression of miR155 successfully (Fig.2).

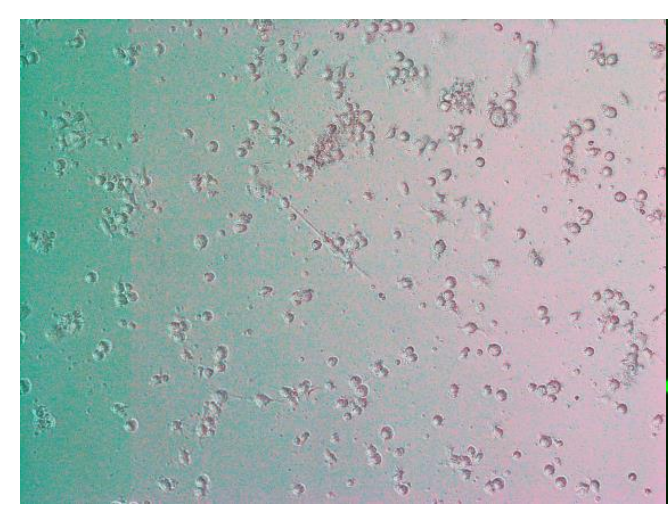

A

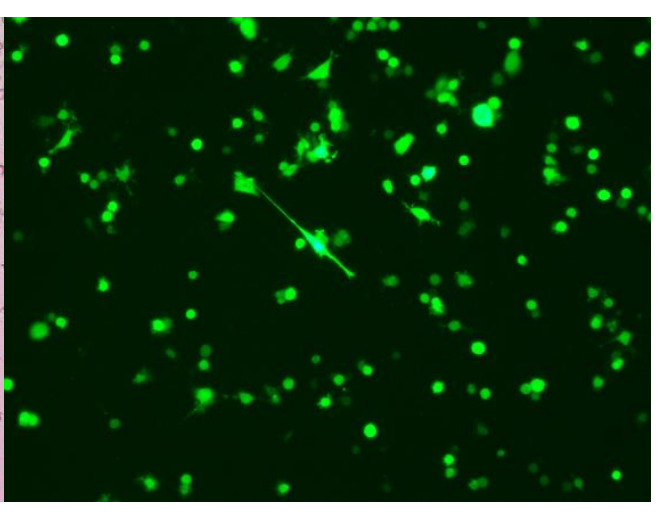

B

Fig1 (A) was taken under normal light observation and the specific dendritic bulges on the surface of DCs were observed. Under inverted fluorescence microscope,(B) showed a transfection efficiency of adenovirus exceeded $85 \%$.
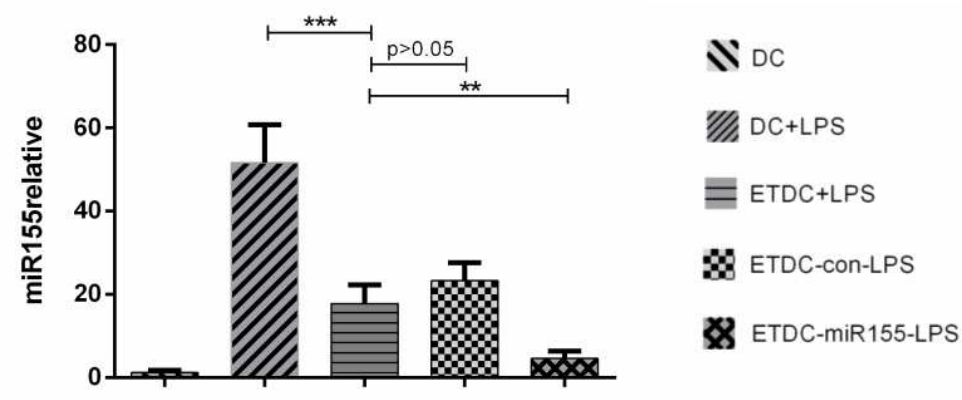

Fig2 Levels of miR155 were standardized to U6 content. MiR155 expression of DCs and ETDCs appaered a significant increasement after stimulated by large dose of LPS. The levels of miR155 in ETDCs were lower than DCs, while the lowest level was measured in miR155--ETDCs. $\quad * *$ Represents $\mathrm{P}<0.01$. ****Represents $\mathrm{P}<0.001$. 
3.2 silencing miR155 attenuated stimulatory capacity of ETDCs to $\mathrm{T}$ cell proliferation

In the mixed lymphocyte reaction,we co-cultured DCs with $\mathrm{T}$ cells in two different ratios of 1:5, 1:10. As shown in Fig3, the ability to induce proliferation of T cells varied in different group.The result revealed that ETDCs has a weaker stimulatory capacity to $\mathrm{T}$ cell proliferation compared to mDCs. What' $\mathrm{s}$ more, silencing miR155 of ETDCs could further attenuated the allostimulatory ability. In all groups, the proliferation of $\mathrm{T}$ cells in 1:5 ratio group was higher with that in 1:10 group.

Fig3

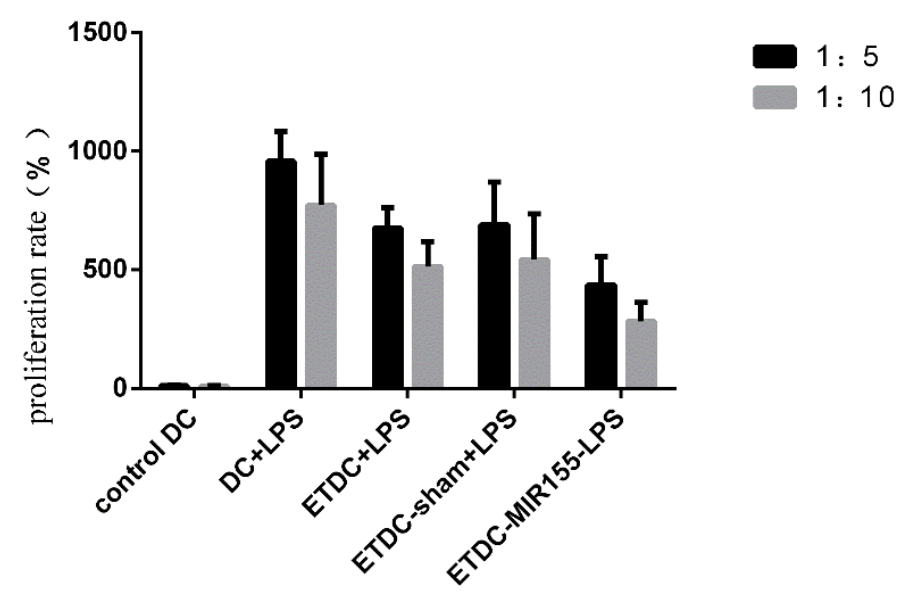

Fig 3 The elevation of optical density (OD) corroborated an increase in T cells quantity when co-cultured with mDCs, ETDCs, ETDCs-con and miR155 ${ }^{-/}$ETDCs, while there was no significant difference co-cultured with imDCs. OD levels were

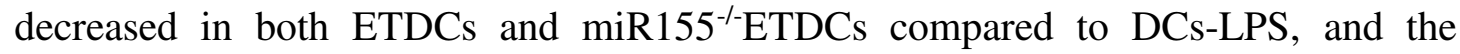
decrease was more prominet in the latter group $(\mathrm{P}<0.05)$. 
3.3 silencing miR155 resulted in lower expression of costimulatory molecules of

DCs

The results of flow cytometry indicated that the levels of MHCII, CD86 and CD11c of DCs increased significantly after LPS stimuli. The expression of costimulatory molecules in ETDCs were lower than mDCs, while silencing miR155 could further attenuate the level of MHCII,CD86 and CD11c. Such a trend suggested that ETDCs performant more close to immature DCs and silencing miR155 could deepen this trend. 
Fig4
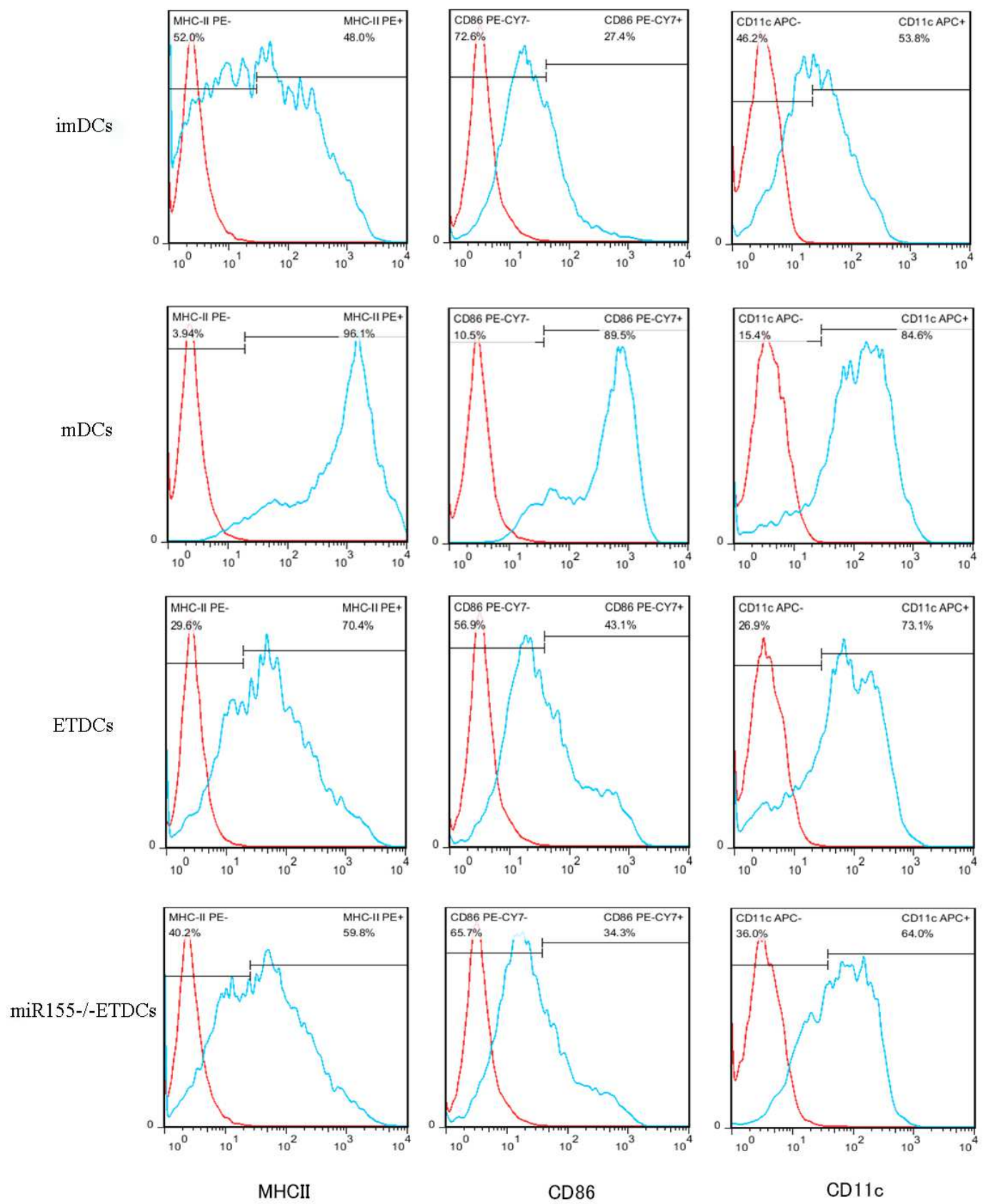

Fig4 Flow cytometry analysis of surface costimulatory factors of DCs, imDCs, mDCs, ETDCs and miR155 ${ }^{-/}$ETDCs were harvested and then analyzed by flow cytometry. The expression of MHCII, CD86 and CD11c in imDCs were $48.0 \%, 27.4 \%$ and $53.8 \%$ 
respectively. These expression level in mDCs increased significantly and reached $96.1 \%, 89.5 \%, 84.6 \%$ after LPS stimuli. The increase of surface costimulatory factors was also found in ETDCs $(70.4 \%, 43.1 \%$ and $73.1 \%$, respectively), but it was significantly lower than that of mDCs $\left(\chi^{2}\right.$ values were $56.47,83.78$ and 23.29, respectively, $\mathrm{P}<0.01$ ). The expression of MHCII,CD86 and CD11c in miR155 ${ }^{-/}$ETDCs were $59.8 \%, 34.3 \%$ and $64.0 \%$, lower than $\operatorname{ETDCs}\left(\chi^{2}\right.$ values were $24.73,16.32$ and 19.21, respectively, $\mathrm{P}<0.01)$.

3.4 silencing miR155 expression inhibited the expression of TNF- $\alpha$ but elevated IL-10 expressionin ETDCs

ELISA results revealed that LPS stimuli could effectively increase cytokine secretion of DCs as there was a significant increase secretion of both TNF- $\alpha$ and IL-10 in supernatant of DCs after LPS stimuli.In the culture supernatant of ETDCs and miR155 ${ }^{-/}$ETDCs ,we found a lower level of TNF- $\alpha$ as well as higher level of IL-10 concentration compared to mDCs after LPS stimuli (Fig.5a,5b). Besides, the highest level of IL-10 and lowest level of TNF- $\alpha$ were observed in miR155 ${ }^{-/}$ETDCs.

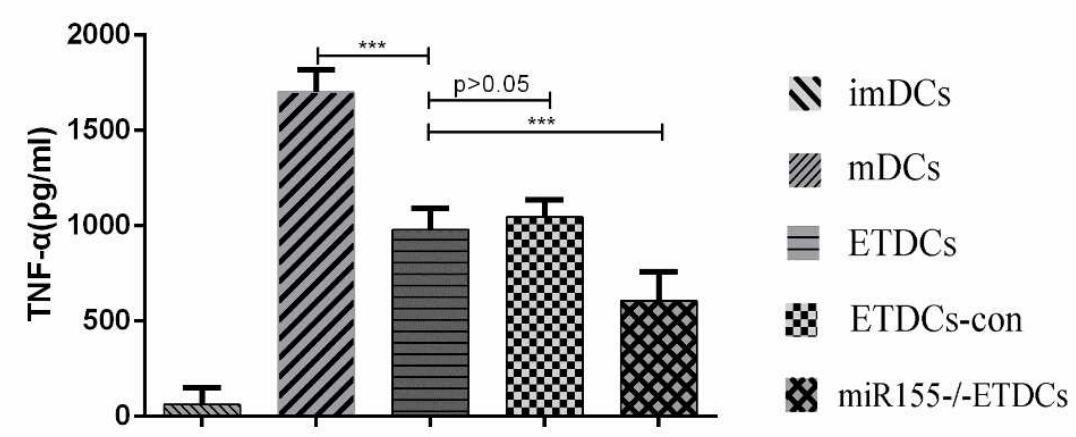

Fig5a 


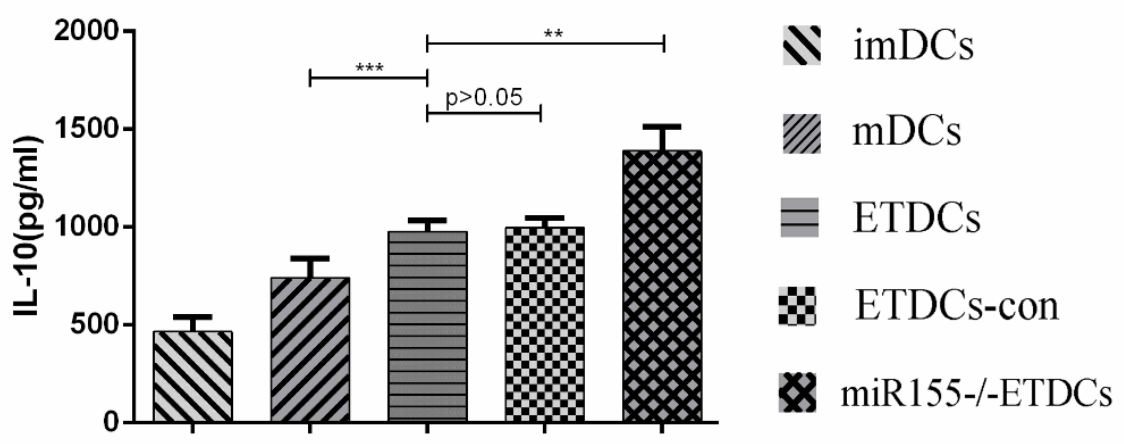

\section{Fig5b}

Fig5a showed that the concentration of TNF- $\alpha$ in the supernatant of mDCs increased markedly. Compared to mDCs, the levels of TNF- $\alpha$ in ETDCs and ETDCscon decreased while the TNF- $\alpha$ concentration in the supernatant of miR $155^{-/}$ETDCs was lowest. Fig5b showed a elevation of IL-10 levels in the supernatant of mDCs, ETDCs and ETDCs-con compared to imDCs. The expression of IL-10 in ETDCs and ETDCs-con were higher than that in mDCs while the highest concentration was detected in the miR155 ${ }^{-/}$ETDCs group. $* *$ Represents $\mathrm{P}<0.01$. $* * *$ Represents $\mathrm{P}<$ 0.001

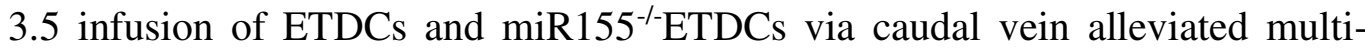
organ injury of murine sepsis model

Sepsis, defined as organ dysfunction caused by a dysregulated host response to infection with the sequential organ ${ }^{1}$, can involve multiple organs. We infused DCs to model mice via tail vein followed by CLP to simulate the pathological injury of sepsis, thereafter the liver, kidney and ileal tissue isolated from each group were observed under microscope staining with H\&E. Our results corroborated severe pathohogical injury in the tissue specimens of sepsis. Lighter pathological injury was detected in the 


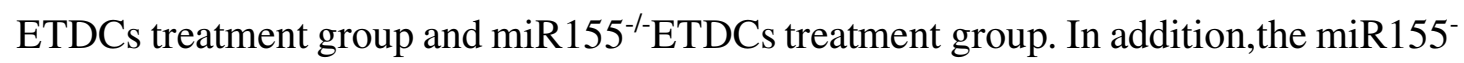

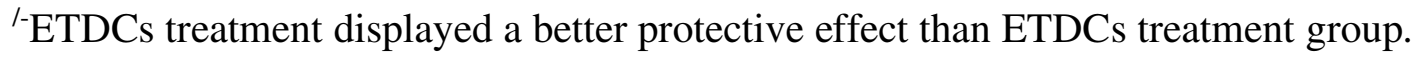

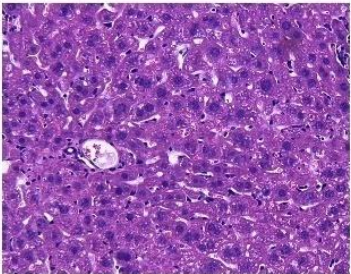

control

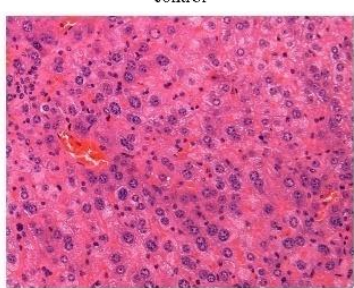

LIDC infusion group

Fig6a

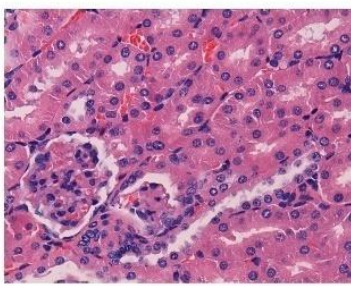

control

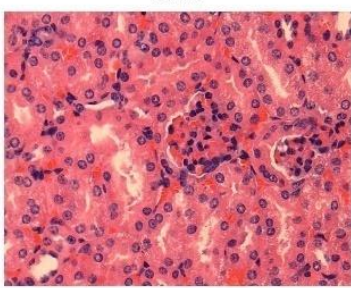

ETDC infusion group

Fig6b

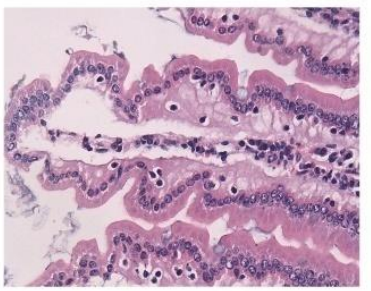

control

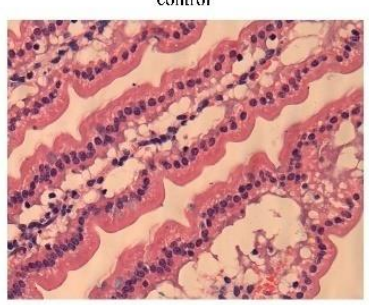

F:TIC infusion group

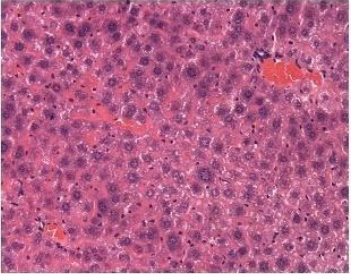

sepsis

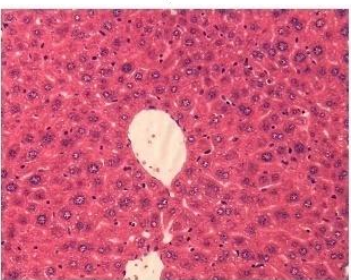

miR 155--FTTC infusion group

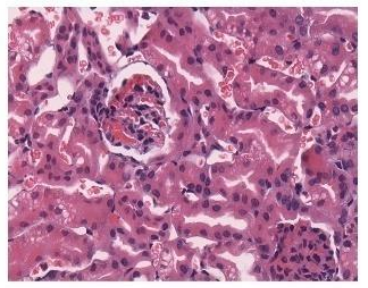

scpsis

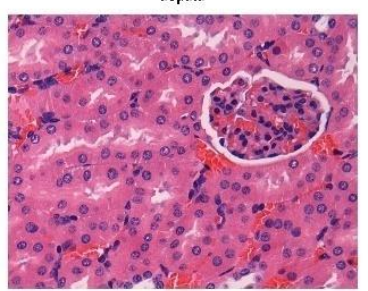

miR155--F.TDC infusion group

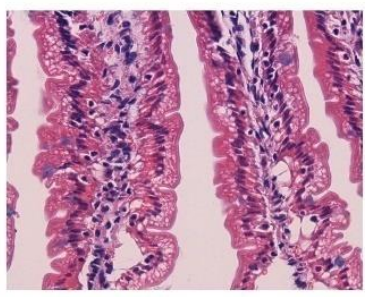

sepsi

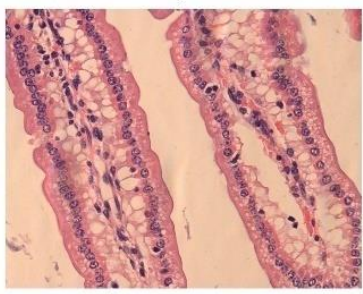

miR 155--LTDC infusion group

Fig6c 
As Fig6a exhibited marked edema, degeneration, disorder of arrangement, and massive hepatocyte necrosis were observed in the liver issue of sepsis group. H\&E staining of renal specimen(Fig6b) manifested edema and necrosis in the renal tubular epithelial cells. The intestinal mucosa indicated edema, vacuolar degeneration, and the top of the villi was damaged(Fig6c). All specimen were accompanied by inflammatory cell infiltration. Both the ETDCs treatment group and the miR $155^{-/}$ETDCs treatment group displayed a significant improvement of these pathological change, while the miR155 ${ }^{-/}$ETDCs treatment group showed a better pathological condition.

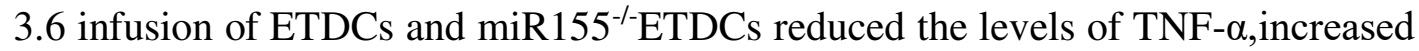
concentration of IL-10

ELISA results revealed that both ETDCs and miR155--ETDCs infusion could alleviate the significant elevation of TNF- $\alpha$ concentration in sepsis group. In addition,the IL-10 levels increased apparently in two treatment groups while the highest level was measured in miR155--ETDCs treatment group.

Fig7
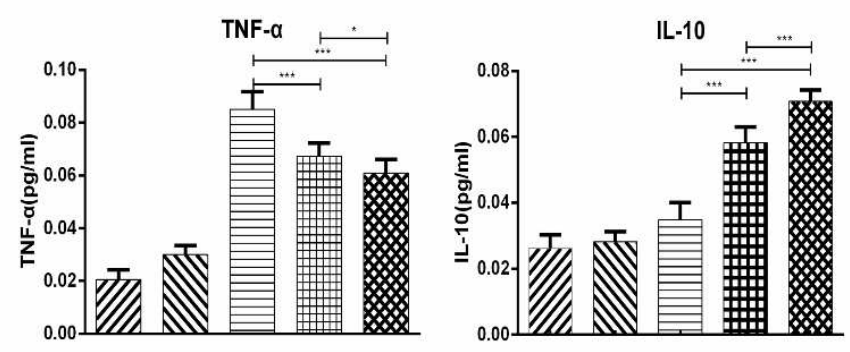

Y. control group

Nish-opcrated group

$=$ sepsis model group

\# ETDCs treatment group

8 miR155--ETDCs treatment group

ELISA results (Fig 8) approved significant growths of TNF- $\alpha(0.085 \pm 0.007 \mathrm{pg} / \mathrm{ml})$ and IL-10(0.036 $\pm 0.005 \mathrm{pg} / \mathrm{ml})$ concentrations in sepsis model serum.Prominet decrease 
of TNF- $\alpha$ levels and increase of IL-10 levels were observed in ETDCs infusion $(0.067 \pm 0.005 \mathrm{pg} / \mathrm{ml}, 0.0582 \pm 0.005 \mathrm{pg} / \mathrm{ml})$ and $\mathrm{miR} 155^{-/}$ETDCs infusion $(0.061 \pm 0.005 \mathrm{pg} / \mathrm{ml}, 0.071 \pm 0.004 \mathrm{pg} / \mathrm{ml})$ groups. The lowest TNF- $\alpha$ and highest IL-10 concentration were detected in the miR $155^{-/}$ETDCs treatment group.

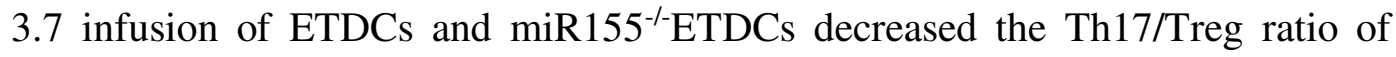
sepsis murine model

The imbalance ratio of Treg and helper T lymphocytes 17 (Th17) will affect the progress of interactions and is related to the occurrence and development of sepsis ${ }^{21}$ and other diseases ${ }^{22,23}$.The results of flow cytometry manifested a high ratio of Th17 /Treg in sepsis murine.Both ETDCs and miR155--ETDCs infusion displayed a ability to attenuate Th17/Treg ratio while the Th17/Treg ratio was lowest in the miR155/ETDCs treatment group.
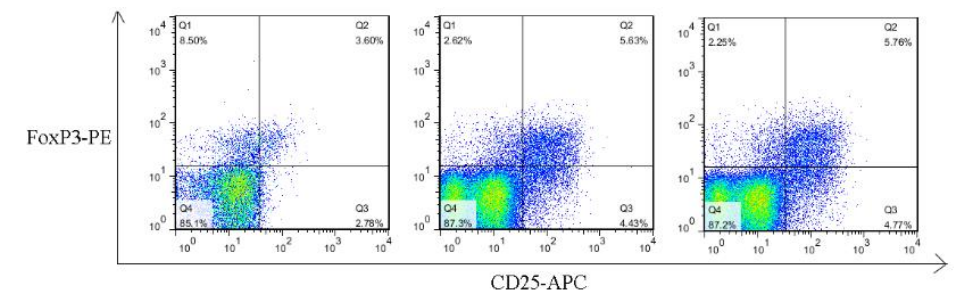

Fig8
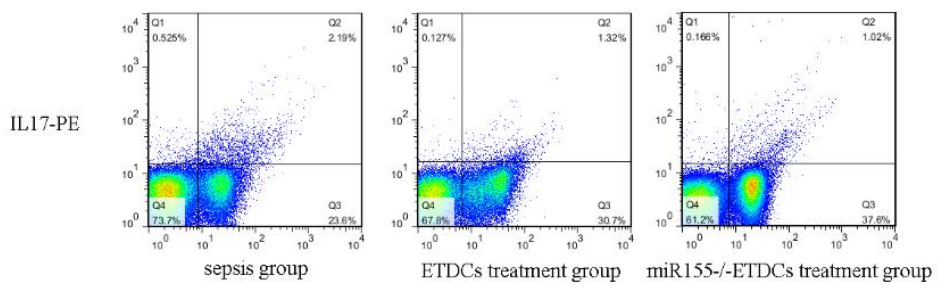

Fig 8 The Treg and Th17 in the spleens of control mice were $0.887 \%$ and $0.59 \%$, respectively.Treg and Th17 in sepsis mice are $3.60 \%$ and $2.19 \%$ respectively and the ratio of Th17/Treg is $60.8 \%$. The Th17/Treg ratio of ETDCs treatment group and 
miR155 ${ }^{-/}$ETDCs treatment group are $23.4 \%$ and $17.7 \%$. The difference between two treatment groups has statistical significance $(\mathrm{P}<0.05)$.

\section{Discussion}

Sepsis can lead to immune system dysfunction, and eventually result in multiple organ failure and septic shock ${ }^{24,25}$. Due to the specific mechanism of sepsis has not been fully elucidated, a effective treatment of sepsis is still lacking. It is believed that the activation of mass immune cells and the inflammatory factor storm formed by the massive secretion of cytokines play an important role in the early onset of sepsis ${ }^{24}$. DC, the only professional antigen presenting cell that can activate the initial $\mathrm{T}$ lymphocyte activation, not only play a pivotal role in regulating immune response or immune tolerance but also exhibit important application value in cell adoptive immunotherapy ${ }^{8,26}$. Predictably, the research of DC targeting immunomodulatory therapy is emerging as a new direction for the treatment of sepsis and hence has a wide prospect. Endotoxin tolerance, as a self-protective mechanism against the inflammatory response induced by lipopolysaccharides, can be utilized as a preventive treatment against endotoxemia ${ }^{27}$.Our previous studies confirmed that endotoxin tolerance manifested a protective role in mice with acute liver failure, which can improve survival rate and alleviate liver pathological injuries ${ }^{10}$.It is reported that ETDCs have a therapeutic effect on autoimmune cerebrospinal myelitis ${ }^{12}$ and tracheitis ${ }^{13}$, but there is little research on its application in sepsis.

As the initiating factor of inflammatory response, the concentration of TNF- $\alpha$ in 
the blood of sepsis patients was remarkblely increased ${ }^{28,29}$. IL-10 is a class of antiinflammatory factors secreted by various immune cells, can affect expression of various cytokines and inflammatory mediators ${ }^{30}$.The results of vitro experiments demonstrated that the level of TNF- $\alpha$ in the supernatant of ETDCs and miR155 ${ }^{-/}$ETDCs were lower than that of mDCs after high-dose lipopolysaccharide stimuli while the level of IL-10 increased siginificantly.

The expression of costimulatory molecules of ETDCs and miR $155^{-/}$ETDCs decreased compared to $\mathrm{mDCs}$, suggesting a transition to immature phenotype accompanied by weakened ability to stimulate allogeneic mice spleen T lymphocytes.In addition,the silencing of miR155 in ETDCs could furthur the transform to immature status.We speculate that inhibit the expression of miR155 could deepen the state of endotoxin tolerance.

CLP, considered as the gold standard model, is the most commonly used model of sepsis $^{31}$. After we performed CLP on mice, H\&E staining exhibited a large amount of inflammatory cell infiltration in the liver, kidney and ileum tissues of the mice, accompanied by cell edema and necrosis. These disorder corroborated the successful establishiment of sepsis model. Our results comfirmed that both ETDCs and miR155 1 ETDCs showed a protection on sepsis model mice while miR $155^{-/}$ETDCs is superior to ETDCs.

ELISA results showed that miR $155^{-/}$ETDCs treatment group had the highest serum IL-10 concentration as well as the lowest TNF- $\alpha$ concentration while flow cytometry analysis indicated the miR155 ${ }^{-/}$ETDCs treatment group gained the lowest Treg/Th17 
ratio. Treg and helper $\mathrm{T}$ lymphocytes 17 (Th17) are two types of $\mathrm{CD}^{+} \mathrm{T}$ cells that are important to immune responses.Tregs are associated with maintaining immune tolerance and can suppress excessive immune responses ${ }^{32}$.Th17 can stimulate the secretion of various inflammatory chemokines and pro-inflammatory factors ${ }^{33}$, and are associated with autoimmune diseases such as rheumatoid arthritis and multiple sclerosis $^{34}$. The imbalance between Treg and Th17 can lead to inflammatory process exaceration and has been reported to involve the occurrence and development of $\operatorname{sepsis}^{21}$ and various other diseases ${ }^{22,23}$. As a negative immunoregulatory factor,IL-10 can promote the differentiation of primitive $\mathrm{T}$ cells into Treg while Treg can inhibit the maturation of DC and induce the generation of regulatory dendritic cells(DCreg $)^{35}$. DCreg can reduce pro-inflammatory cytokines such as TNF- $\alpha$, negatively regulate the immune response, and alleviate the damages caused by excessive immunity.

In conclusion, according to our experimental results, we speculate that miR155 。 to negatively regulate immune response than ETDCs. The protective effects of ETDCs and miR $155^{-/}$ETDCs in the early stage of sepsis may achieved by altering the concentration of mouse cytokines to affect $\mathrm{T}$ cell differentiation and exert negative immune regulation. The regulation of miR155 may provides a novel approach of adoptive immunotherapy for sepsis.

\section{Ethics approval and consent to participate:}

All protocols were authorized by the Animal Ethics Committee of Wenzhou Medical University. 


\section{Consent for publication:}

Not applicable.

Availability of data and materials: The data used to support the findings of this study are available from the corresponding author upon reasonable request.

Competing interests: The authors declare that they have no competing interests.

Funding: This work was supported by the Science and Technology Planned Projects of Wenzhou City (No.2017Y0999, No. 2018Y0330).

Authors' contributions : Min Yang and Chao-Chen Hou and Yan-Yan Yang performed the experiments and analyzed the data; Shan-Shan Li,De-Yong Kong and You-Ran Chen interpreted the data and drafted the manuscript; Hui-Fang Zhang and Ming-Qin Lu designed the project and reviewed and edited the manuscript. The author(s) read and approved the final manuscript.

\section{Acknowledgements:}

Not applicable.

\section{Abbreviations:}


DCs:dendritic cells

ETDCs:endotoxin tolerant dendritic cells

miR155: microRNA-155

TNF- $\alpha$ :tumor necrosis factor-alpha

IL-10:interleukin-10

MOI: appropriate multiplicity of infection

CLP: cacal ligation puncture

\section{References}

1. Singer M, Deutschman CS, Seymour CW, et al. The Third International Consensus Definitions for Sepsis and Septic Shock (Sepsis-3). JAMA. 2016;315(8):801-810. doi:10.1001/jama.2016.0287

2. Rubio I, Osuchowski MF, Shankar-Hari M, et al. Current gaps in sepsis immunology: new opportunities for translational research. Lancet Infect Dis. Published online October 17, 2019. doi:10.1016/S1473-3099(19)30567-5

3. Vincent J-L, Marshall JC, Namendys-Silva SA, et al. Assessment of the worldwide burden of critical illness: the intensive care over nations (ICON) audit. Lancet Respir Med. 2014;2(5):380-386. doi:10.1016/S2213-2600(14)70061-X

4. Shankar-Hari M, Ambler M, Mahalingasivam V, Jones A, Rowan K, Rubenfeld GD. Evidence for a causal link between sepsis and long-term mortality: a systematic review of epidemiologic studies. Crit Care Lond Engl. 2016;20:101. doi:10.1186/s13054-016-1276-7 
5. Pfortmueller CA, Meisel C, Fux M, Schefold JC. Assessment of immune organ dysfunction in critical illness: utility of innate immune response markers. Intensive Care Med Exp. 2017;5(1):49. doi:10.1186/s40635-017-0163-0

6. van der Poll T, van Deventer SJ. Cytokines and anticytokines in the pathogenesis of sepsis. Infect Dis Clin North Am. 1999;13(2):413-426, ix. doi:10.1016/s0891-5520(05)70083-0

7. Sabado RL, Balan S, Bhardwaj N. Dendritic cell-based immunotherapy. Cell Res. 2017;27(1):74-95. doi:10.1038/cr.2016.157

8. Subbotin VM. Dendritic cell-based cancer immunotherapy: the stagnant approach and a theoretical solution. Drug Discov Today. 2014;19(7):834-837. doi:10.1016/j.drudis.2014.02.008

9. Biswas SK, Lopez-Collazo E. Endotoxin tolerance: new mechanisms, molecules and clinical significance. Trends Immunol. 2009;30(10):475-487. doi:10.1016/j.it.2009.07.009

10. Yang N-B, Ni S-L, Li S-S, Zhang S-N, Hu D-P, Lu M-Q. Endotoxin tolerance alleviates experimental acute liver failure via inhibition of high mobility group box 1 . Int J Clin Exp Pathol. 2015;8(8):9062-9071.

11. Zhang S-N, Yang N-B, Ni S-L, et al. Splenic CD11c(low)CD45RB(high) dendritic cells derived from endotoxin-tolerant mice attenuate experimental acute liver failure. Sci Rep. 2016;6:33206. doi:10.1038/srep33206

12. Zhou F, Ciric B, Zhang G-X, Rostami A. Immunotherapy using lipopolysaccharide-stimulated bone marrow-derived dendritic cells to treat 
experimental autoimmune encephalomyelitis. Clin Exp Immunol. 2014;178(3):447458. doi:10.1111/cei.12440

13. Wong T-H, Chen H-A, Gau R-J, Yen J-H, Suen J-L. Heme Oxygenase-1Expressing Dendritic Cells Promote Foxp3+ Regulatory T Cell Differentiation and Induce Less Severe Airway Inflammation in Murine Models. PloS One. 2016;11(12):e0168919. doi:10.1371/journal.pone.0168919

14. Usui $Y$, Takeuchi $M$, Hattori $T$, et al. Suppression of experimental autoimmune uveoretinitis by regulatory dendritic cells in mice. Arch Ophthalmol Chic Ill 1960. 2009;127(4):514-519. doi:10.1001/archophthalmol.2009.34

15. Chen J-Q, Papp G, Szodoray P, Zeher M. The role of microRNAs in the pathogenesis of autoimmune diseases. Autoimmun Rev. 2016;15(12):1171-1180. doi:10.1016/j.autrev.2016.09.003

16. Creugny A, Fender A, Pfeffer S. Regulation of primary microRNA processing. FEBS Lett. 2018;592(12):1980-1996. doi:10.1002/1873-3468.13067

17. Elton TS, Selemon H, Elton SM, Parinandi NL. Regulation of the MIR155 host gene in physiological and pathological processes. Gene. 2013;532(1):1-12. doi:10.1016/j.gene.2012.12.009

18. Moffett HF, Novina CD. A small RNA makes a Bic difference. Genome Biol. 2007;8(7):221. doi:10.1186/gb-2007-8-7-221

19. Doxaki C, Kampranis SC, Eliopoulos AG, Spilianakis C, Tsatsanis C. Coordinated Regulation of miR-155 and miR-146a Genes during Induction of 
Endotoxin Tolerance in Macrophages. J Immunol Baltim Md 1950. 2015;195(12):5750-5761. doi:10.4049/jimmunol.1500615

20. Miller G, Lahrs S, Shah AB, DeMatteo RP. Optimization of dendritic cell maturation and gene transfer by recombinant adenovirus. Cancer Immunol Immunother CII. 2003;52(6):347-358. doi:10.1007/s00262-003-0379-6

21. Guo J, Tao W, Tang D, Zhang J. Th17/regulatory T cell imbalance in sepsis patients with multiple organ dysfunction syndrome: attenuated by high-volume hemofiltration. Int J Artif Organs. 2017;40(11):607-614. doi:10.5301/ijao.5000625

22. Abdolahi M, Yavari P, Honarvar NM, Bitarafan S, Mahmoudi M, SaboorYaraghi AA. Molecular Mechanisms of the Action of Vitamin A in Th17/Treg Axis in Multiple Sclerosis. J Mol Neurosci MN. 2015;57(4):605-613. doi:10.1007/s12031-0150643-1

23. Zhang L, Wan F, Song J, et al. Imbalance Between Th17 Cells and Regulatory T Cells During Monophasic Experimental Autoimmune Uveitis. Inflammation. 2016;39(1):113-122. doi:10.1007/s10753-015-0229-7

24. Chousterman BG, Swirski FK, Weber GF. Cytokine storm and sepsis disease pathogenesis. Semin Immunopathol. 2017;39(5):517-528. doi:10.1007/s00281-017$0639-8$

25. Morgan RW, Fitzgerald JC, Weiss SL, Nadkarni VM, Sutton RM, Berg RA. Sepsis-associated in-hospital cardiac arrest: Epidemiology, pathophysiology, and potential therapies. J Crit Care. 2017;40:128-135. doi:10.1016/j.jcrc.2017.03.023 
26. Banchereau J, Steinman RM. Dendritic cells and the control of immunity. Nature. 1998;392(6673):245-252. doi:10.1038/32588

27. Saturnino SF, Prado RO, Cunha-Melo JR, Andrade MV. Endotoxin tolerance and cross-tolerance in mast cells involves TLR4, TLR2 and FcepsilonR1 interactions and SOCS expression: perspectives on immunomodulation in infectious and allergic diseases. BMC Infect Dis. 2010;10:240. doi:10.1186/1471-2334-10-240

28. Feezor RJ, Oberholzer C, Baker HV, et al. Molecular characterization of the acute inflammatory response to infections with gram-negative versus gram-positive bacteria. Infect Immun. 2003;71(10):5803-5813. doi:10.1128/iai.71.10.58035813.2003

29. Acar L, Atalan N, Karagedik EH, Ergen A. Tumour Necrosis Factor-alpha and Nuclear Factor-kappa B Gene Variants in Sepsis. Balk Med J. 2018;35(1):30-35. doi:10.4274/balkanmedj.2017.0246

30. Fiorentino DF, Zlotnik A, Mosmann TR, Howard M, O'Garra A. IL-10 inhibits cytokine production by activated macrophages. J Immunol Baltim Md 1950. 1991;147(11):3815-3822.

31. Dejager L, Pinheiro I, Dejonckheere E, Libert C. Cecal ligation and puncture: the gold standard model for polymicrobial sepsis? Trends Microbiol. 2011;19(4):198208. doi:10.1016/j.tim.2011.01.001

32. Sakaguchi S, Yamaguchi T, Nomura T, Ono M. Regulatory T cells and immune tolerance. Cell. 2008;133(5):775-787. doi:10.1016/j.cell.2008.05.009 
33. Kolls JK, Lindén A. Interleukin-17 family members and inflammation. Immunity. 2004;21(4):467-476. doi:10.1016/j.immuni.2004.08.018

34. Yang J, Sundrud MS, Skepner J, Yamagata T. Targeting Th17 cells in autoimmune diseases. Trends Pharmacol Sci. 2014;35(10):493-500. doi:10.1016/j.tips.2014.07.006

35. Matsuda R, Kezuka T, Nishiyama C, et al. Interleukin-10 gene-transfected mature dendritic cells suppress murine experimental autoimmune optic neuritis. Invest Ophthalmol Vis Sci. 2012;53(11):7235-7245. doi:10.1167/iovs.12-10587 


\section{Figures}
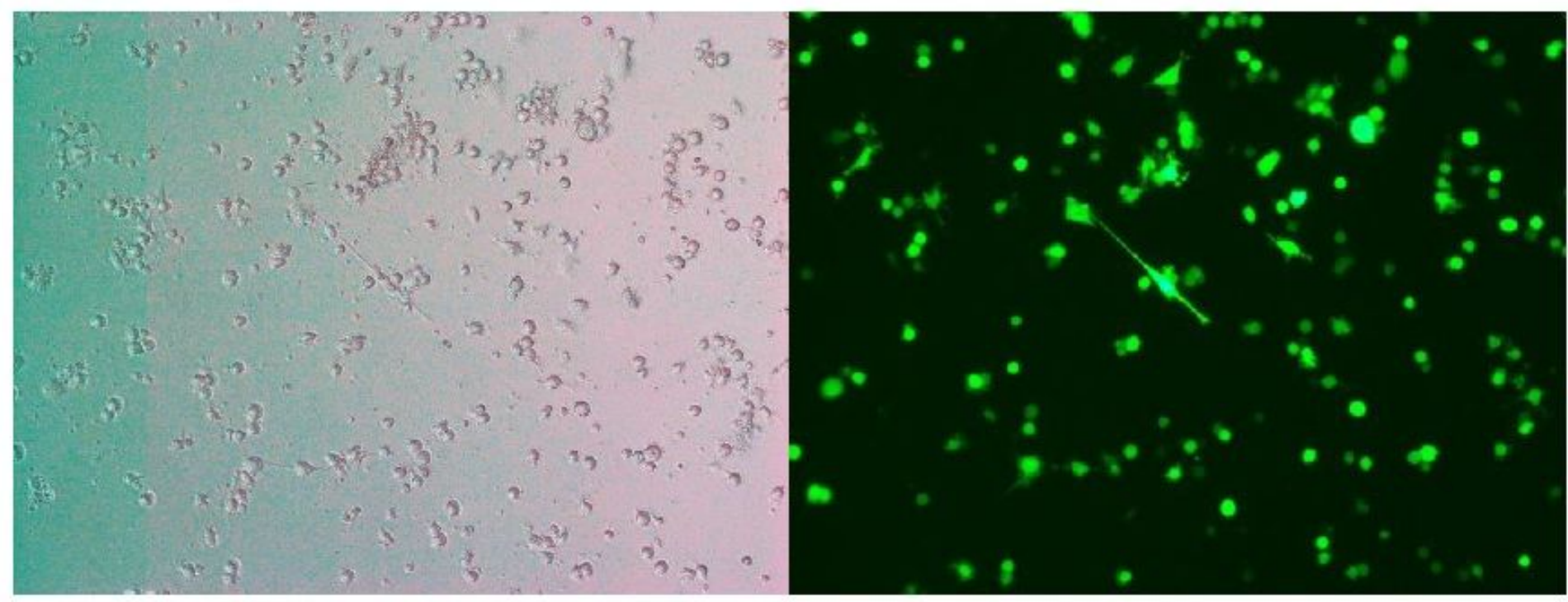

A

B

Figure 1

$\triangle A)$ was taken under normal light observation and the specific dendritic bulges on the surface of DCs were observed. Under inverted fluorescence microscope,(B) showed a transfection efficiency of adenovirus exceeded $85 \%$.

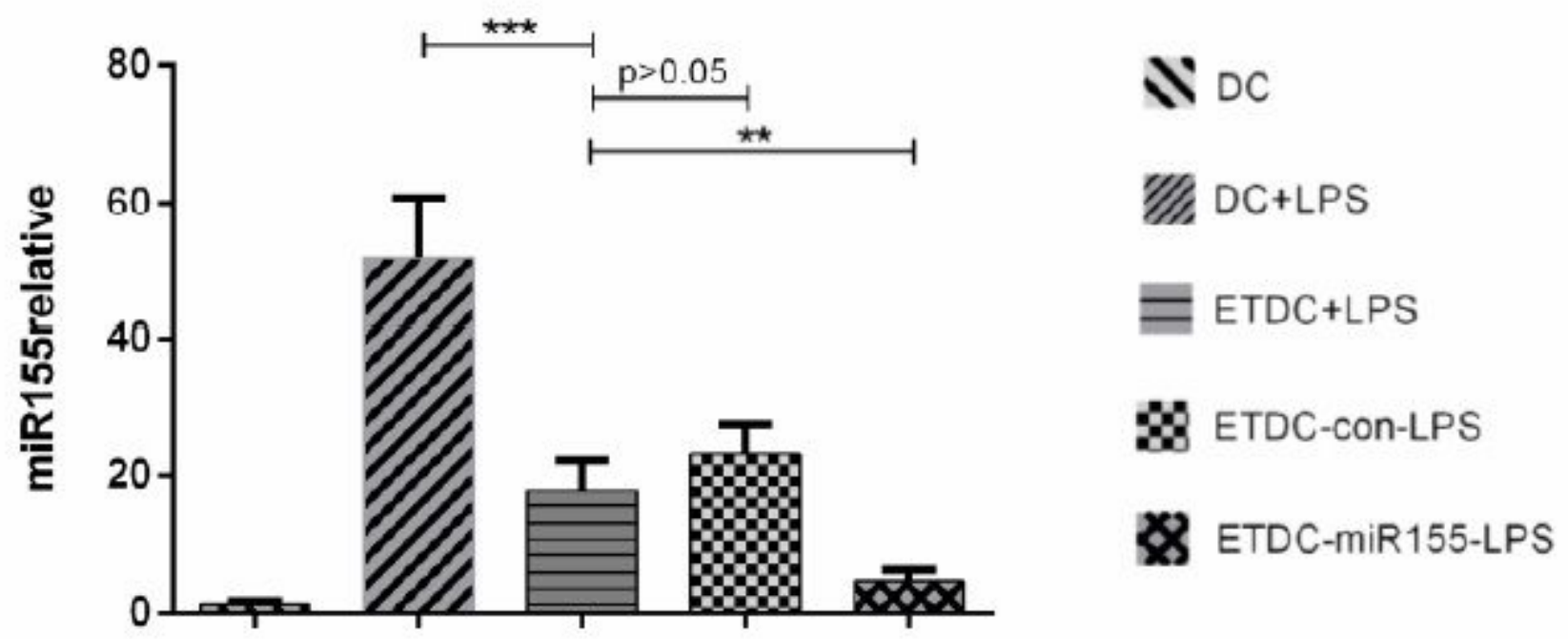

Figure 2

Levels of miR155 were standardized to U6 content. MiR155 expression of DCs and ETDCs appaered a significant increasement after stimulated by large dose of LPS. The levels of miR155 in ETDCs were 
lower than DCs, while the lowest level was measured in miR155-/-ETDCs. **Represents $\mathrm{P}<0.01$. $\star \star *$ Represents $\mathrm{P}<0.001$.

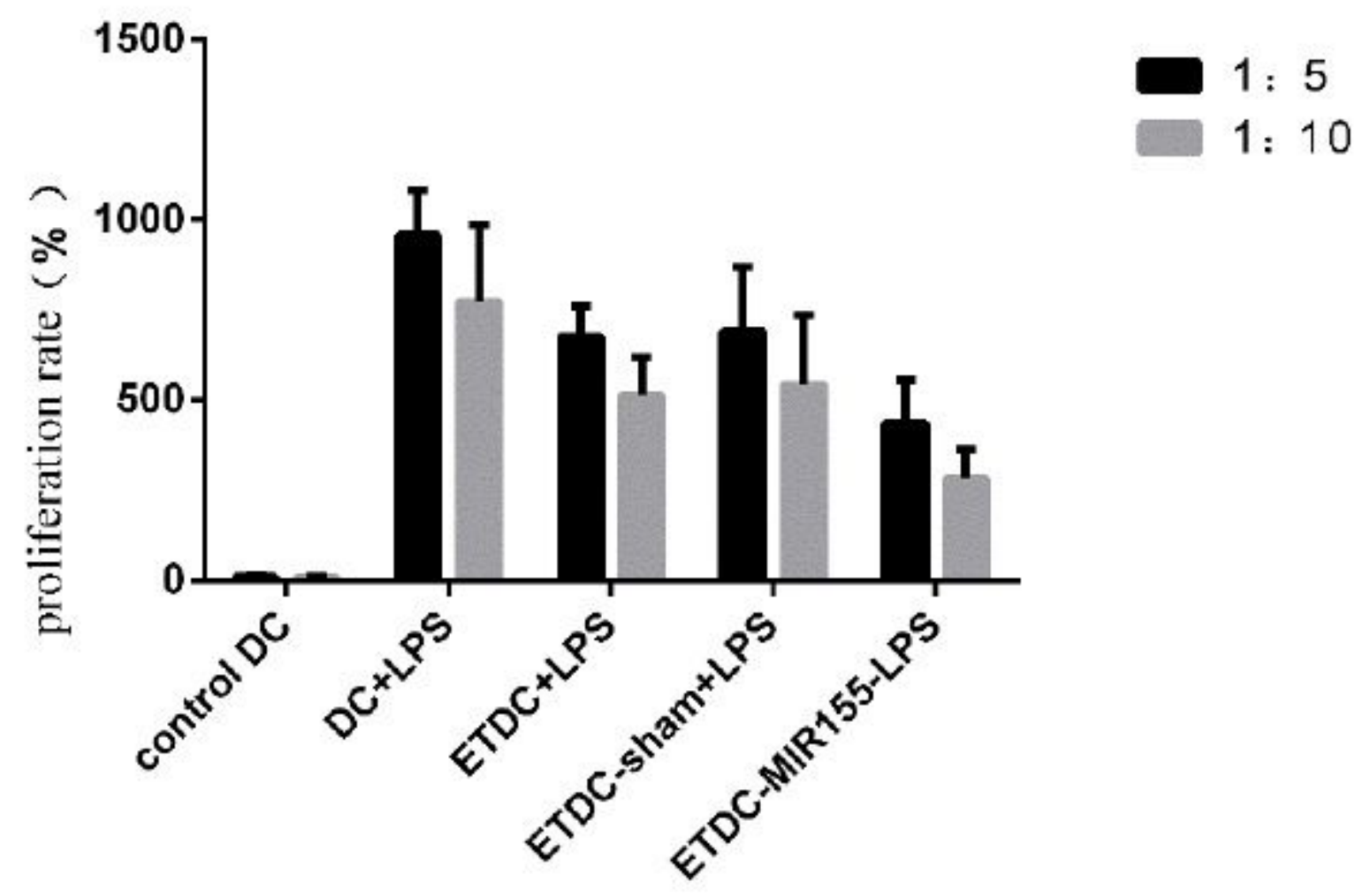

Figure 3

The elevation of optical density (OD) corroborated an increase in T cells quantity when co-cultured with mDCs, ETDCs, ETDCs-con and miR155-/-ETDCs, while there was no significant difference co-cultured with imDCs. OD levels were decreased in both ETDCs and miR155-/-ETDCs compared to DCs-LPS, and the decrease was more prominet in the latter group $(P<0.05)$. 

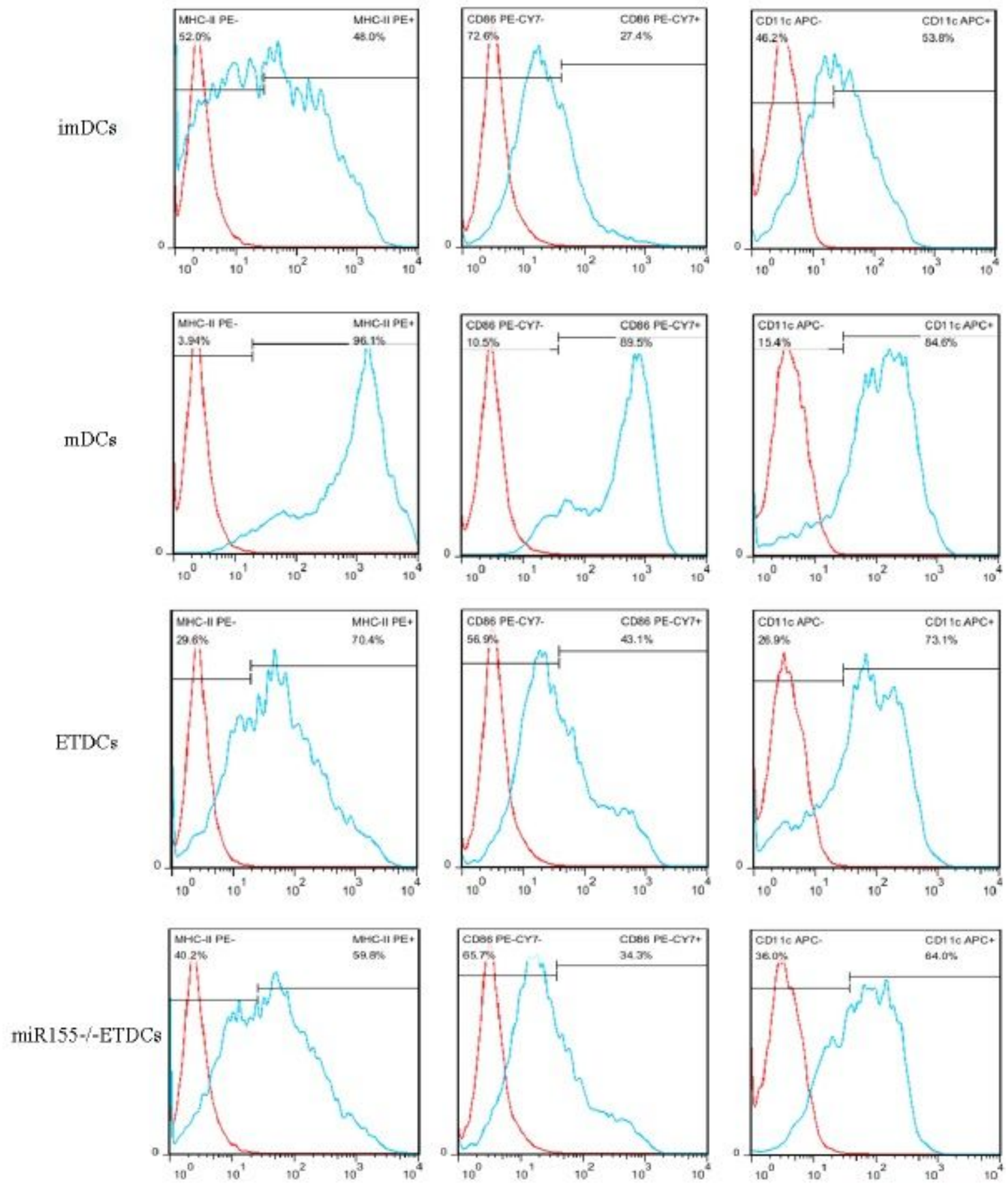

MHCII

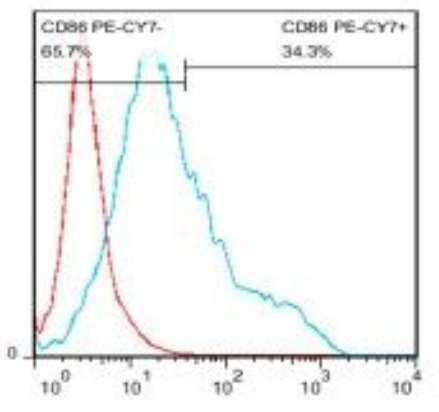

CD86

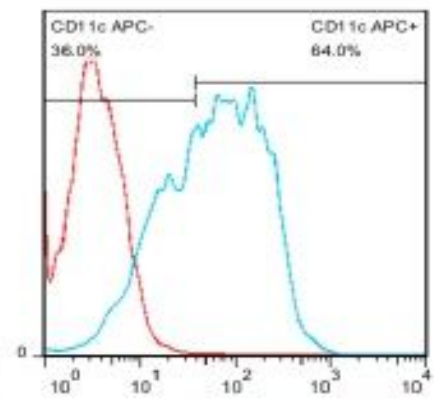

CD11c

\section{Figure 4}

Flow cytometry analysis of surface costimulatory factors of DCs, imDCs, mDCs, ETDCs and miR155-/ETDCs were harvested and then analyzed by flow cytometry. The expression of MHCII, CD86 and CD11C in imDCs were $48.0 \%, 27.4 \%$ and $53.8 \%$ respectively. These expression level in $\mathrm{mDCs}$ increased significantly and reached $96.1 \%, 89.5 \%, 84.6 \%$ after LPS stimuli. The increase of surface costimulatory factors was also found in ETDCs $(70.4 \%, 43.1 \%$ and $73.1 \%$, respectively), but it was significantly lower 
than that of $\mathrm{mDCs}$ ( $\chi 2$ values were $56.47,83.78$ and 23.29 , respectively, $\mathrm{P}<0.01$ ). The expression of MHCII,CD86 and CD11c in miR155-/-ETDCs were 59.8\%,34.3\% and 64.0\%, lower than ETDCs(X2 values were $24.73,16.32$ and 19.21 , respectively, $\mathrm{P}<0.01$ ).

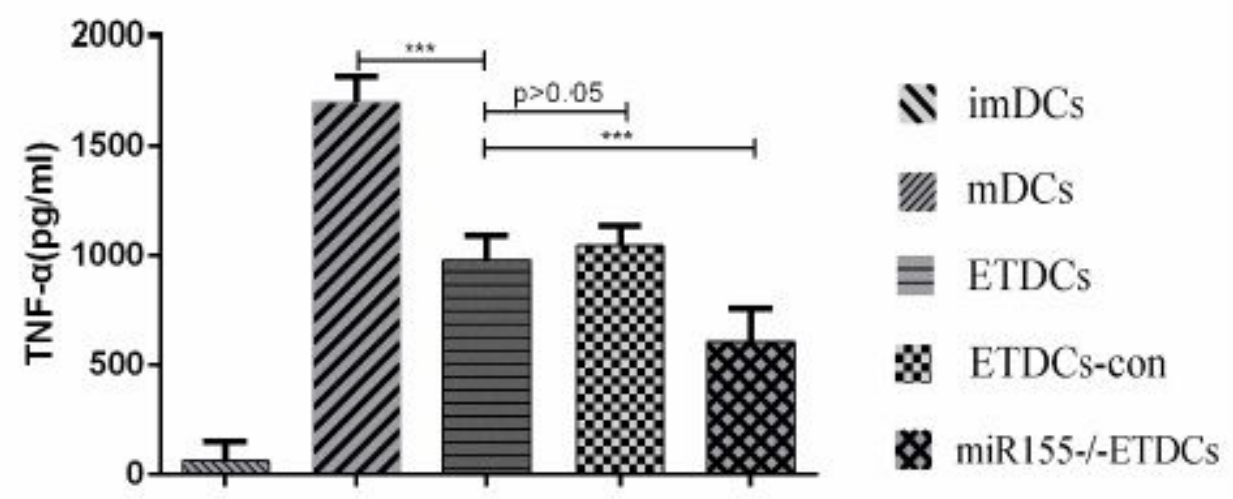

a

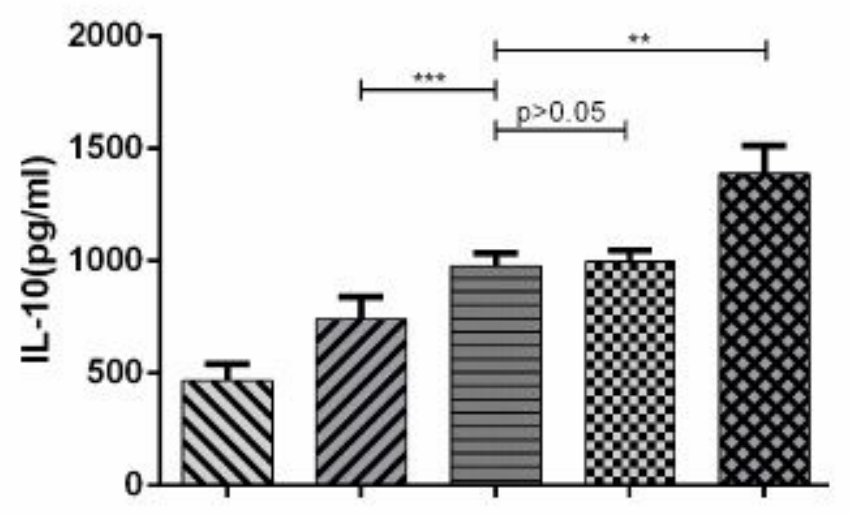

$\mathbb{N}$ imDCs

Wha mDCs

$\equiv \mathrm{ETDCs}$

$\$$ ETDCs-con

$\$$ miR155-/-ETDCs

b

Figure 5

Fig5a showed that the concentration of TNF-a in the supernatant of mDCs increased markedly. Compared to mDCs, the levels of TNF-a in ETDCs and ETDCs-con decreased while the TNF-a concentration in the supernatant of miR155-/-ETDCs was lowest. Fig5b showed a elevation of IL-10 
levels in the supernatant of mDCs, ETDCs and ETDCs-con compared to imDCs. The expression of IL-10 in ETDCs and ETDCs-con were higher than that in $\mathrm{mDC}$ s while the highest concentration was detected in the miR155-/-ETDCs group. **Represents $\mathrm{P}<0.01$. ${ }^{* \star * R e p r e s e n t s ~} \mathrm{P}<0.001$.

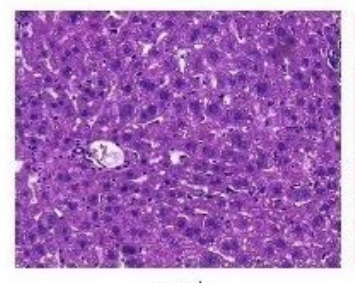

control

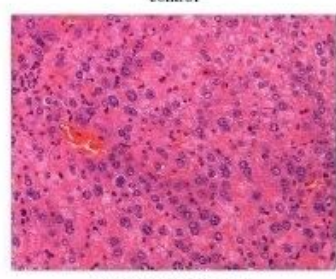

a

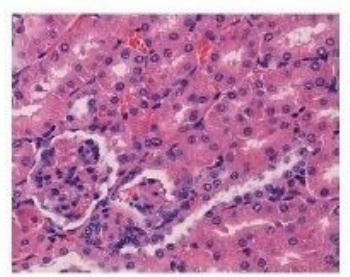

contro]

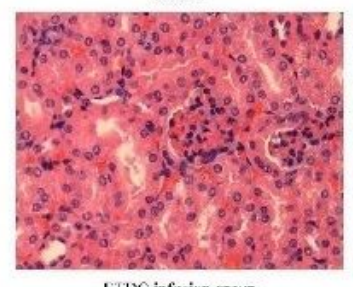

b

ETDC infisisn group
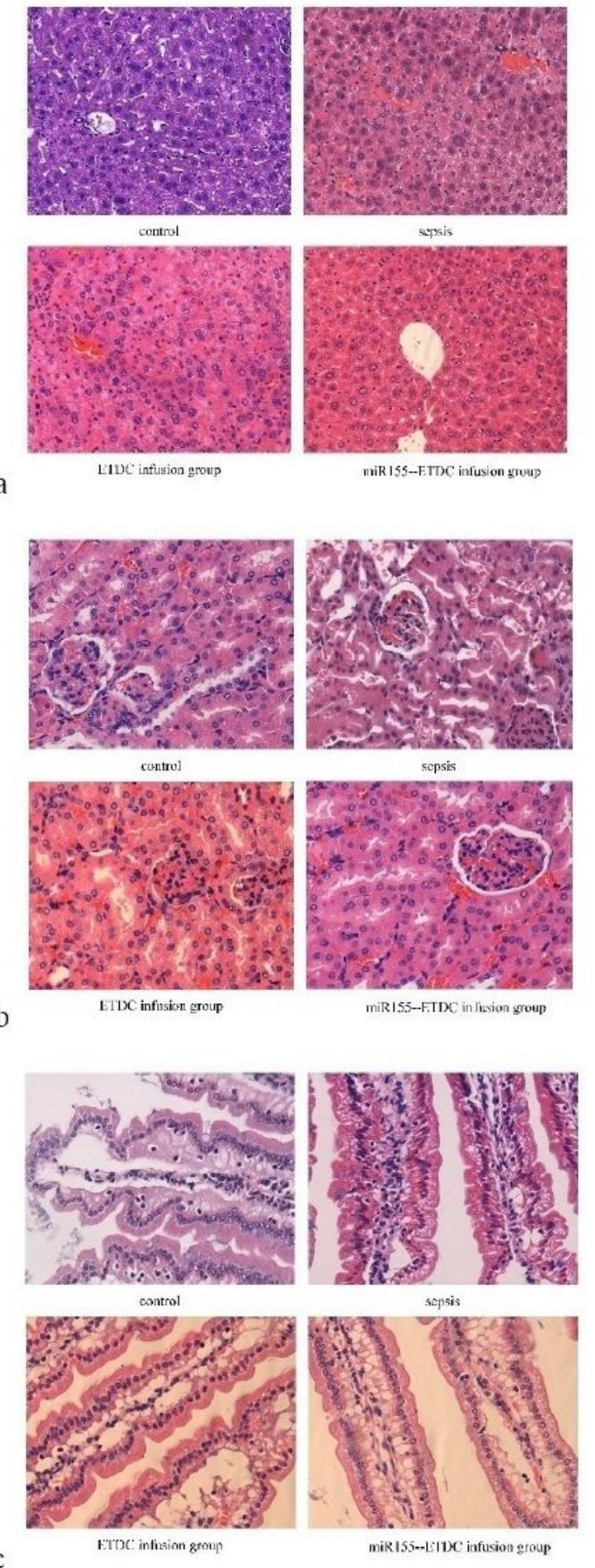

sepsis

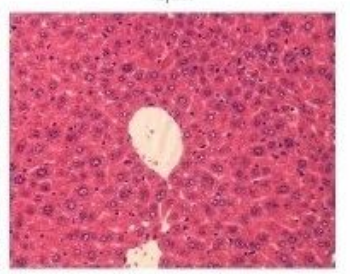

niR 155--FTTC infusion group

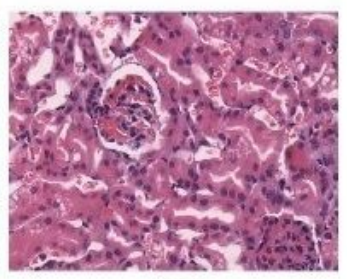

sepsis

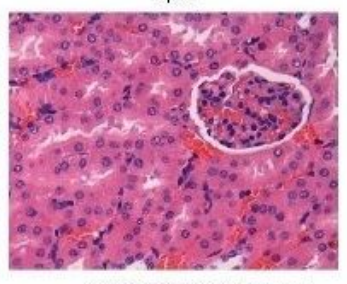

miRI55-F.TDC in litsion yroup

\footnotetext{
(1)
} 
edema and necrosis in the renal tubular epithelial cells. The intestinal mucosa indicated edema, vacuolar degeneration, and the top of the villi was damaged(Fig6c).

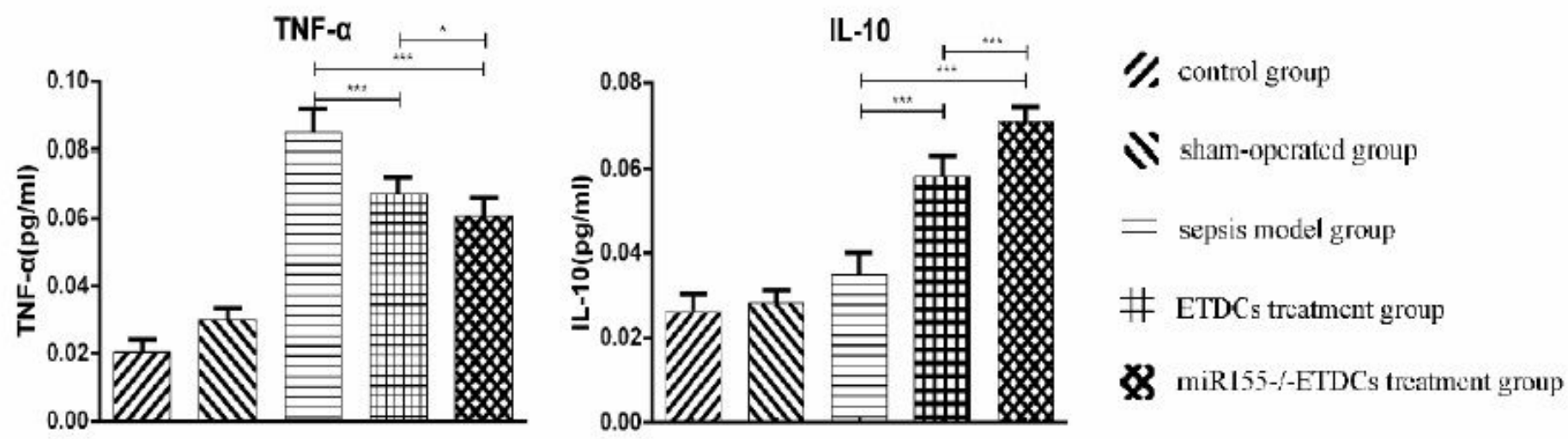

Figure 7

(no caption included)
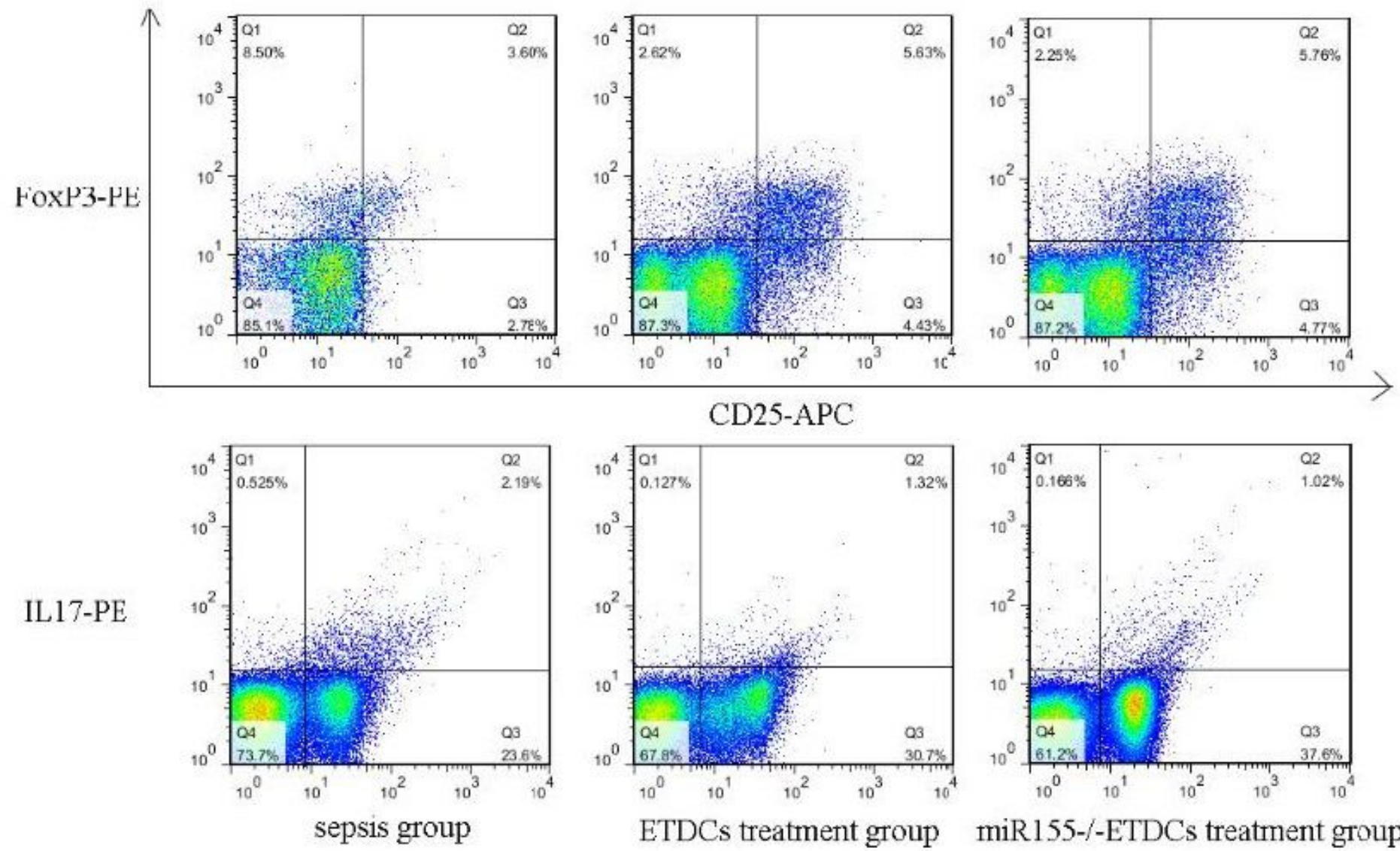

ETDCs treatment group

miR155-/-ETDCs treatment group

\section{Figure 8}

The Treg and Th17 in the spleens of control mice were $0.887 \%$ and $0.59 \%$, respectively.Treg and Th17 in sepsis mice are $3.60 \%$ and $2.19 \%$ respectively and the ratio of Th17/Treg is $60.8 \%$. The Th17/Treg ratio of 
ETDCs treatment group and miR155-/-ETDCs treatment group are $23.4 \%$ and $17.7 \%$. The difference between two treatment groups has statistical significance $(P<0.05)$. 